\title{
Imaging DNA Damage Allows Detection of Preneoplasia in the BALB-neuT Model of Breast Cancer
}

\author{
Bart Cornelissen ${ }^{1}$, Sarah Able ${ }^{1}$, Christiana Kartsonaki ${ }^{1}$, Veerle Kersemans ${ }^{1}$, P. Danny Allen ${ }^{1}$, Federica Cavallo ${ }^{2}$, \\ Jean-Baptiste Cazier ${ }^{1}$, Manuela Iezzi ${ }^{3}$, James Knight ${ }^{1}$, Ruth Muschel ${ }^{1}$, Sean Smart ${ }^{1}$, and Katherine A. Vallis ${ }^{1}$ \\ ${ }^{I}$ Department of Oncology, CRUK/MRC Oxford Institute for Radiation Oncology, Oxford University, Oxford, United Kingdom; \\ ${ }^{2}$ Molecular Biotechnology Center, University of Turin, Turin, Italy; and ${ }^{3}$ CeSI Foundation, University G. d' Annunzio, Chieti, Italy
}

\begin{abstract}
A prominent feature of many human cancers is oncogene-driven activation of the DNA damage response (DDR) during early tumorigenesis. It has been shown previously that noninvasive imaging of the phosphorylated histone $\mathrm{H} 2 \mathrm{~A}$ variant $\mathrm{H} 2 \mathrm{AX}, \mathrm{yH} 2 \mathrm{AX}$, a DNA damage signaling protein, is possible using ${ }^{111} \mathrm{In}$-labeled anti-yH2AX antibody conjugated to the cell-penetrating peptide transactivator of transcription (TAT). The purpose of this study was to investigate whether ${ }^{111} \mathrm{In}$ anti-yH2AX-TAT detects the DDR during mammary oncogenesis in BALB-neuT mice. Methods: Mammary fat pads from BALB-neuT and wild-type mice (age, 40-106 d) were immunostained for $\mathrm{yH} 2 \mathrm{AX}$. ${ }^{111}$ In-anti-yH2AX-TAT or a control probe was administered intravenously to BALB-neuT mice. SPECT was performed weekly and compared with tumor detection using palpation and dynamic contrastenhanced MR imaging. Results: $\mathrm{yH} 2 \mathrm{AX}$ expression was elevated in hyperplastic lesions in the mammary fat pads of BALB-neuT mice aged 76-106 d, compared with normal fat pads from younger mice and carcinomas from older mice $(13.5 \pm 1.2 \mathrm{yH} 2 \mathrm{AX}$ foci/cell vs. $5.2 \pm$ $1.5[P<0.05]$ and $3.4 \pm 1.1[P<0.001]$, respectively). Serial SPECT imaging revealed a 2.5 -fold increase in ${ }^{111} \mathrm{In}$-anti-yH2AX-TAT accumulation in the mammary fat pads of mice aged 76-106 d, compared with control probe $(P=0.01)$. The median time to detection of neoplastic lesions by ${ }^{111} \mathrm{In}$-anti-yH2AX-TAT (defined as $>5 \%$ injected dose per gram of tissue) was $96 \mathrm{~d}$, compared with 120 and $131 \mathrm{~d}$ for dynamic contrast-enhanced MR imaging and palpation, respectively $(P<0.001)$. Conclusion: DDR imaging using ${ }^{111}$ In-anti-yH2AXTAT identified mammary tumors significantly earlier than MR imaging. Imaging the DDR holds promise for the detection of preneoplasia and as a technique for screening cancer-prone individuals.
\end{abstract}

Key Words: $\mathrm{yH} 2 \mathrm{AX}$; molecular imaging; BALB-NeuT; breast cancer; early diagnosis

J Nucl Med 2014; 55:2026-2031

DOI: 10.2967/jnumed.114.142083

$\mathbf{T}$

he DNA damage response (DDR) is a highly regulated cellular process that follows exposure to ionizing radiation and other genotoxic insults (1). The DDR is activated during the earliest stages of cancer development, when it is triggered by the onset of oncogenic stress. Signaling via the ataxia telangiectasia mu-

Received Apr. 24, 2014; revision accepted Oct. 8, 2014.

For correspondence or reprints contact: Katherine A. Vallis, CRUK/MRC

Oxford Institute for Radiation Oncology, University of Oxford, Old Road

Campus Research Building, Off Roosevelt Dr., Oxford, OX3 7DQ U.K.

E-mail: katherine.vallis@oncology.ox.ac.uk

Published online Nov. 11, 2014.

COPYRIGHT (C) 2014 by the Society of Nuclear Medicine and Molecular Imaging, Inc. tated (ATM)-Chk2-p53 DDR pathway minimizes genomic instability and inhibits cell cycle progression and tumor formation (2). This protective mechanism is eventually overridden, possibly precipitated by mutation of the p53 gene (3). Early reports of DDR activation during bladder, colon, breast, skin, and lung cancer development showed that it was accompanied by the activation of signaling proteins downstream of phosphorylated ATM (pATM), including pChk2 and the phosphorylated histone $\mathrm{H} 2 \mathrm{~A}$ variant $\mathrm{H} 2 \mathrm{AX}$, $\gamma \mathrm{H} 2 \mathrm{AX}(4,5)$. DDR activation has since been observed to occur during the development of many other types of cancer including pancreatic (6), ovarian (7), and hepatocellular carcinomas (8). Given the near-ubiquitous nature of DDR activation during oncogenesis, it has been suggested that markers of DNA replication stress and constitutive DDR activation might distinguish neoplastic growth from normal tissues. This hypothesis has been substantiated by immunohistochemical analyses of human tissues. In one study, markers of DDR activation were observed only in subpopulations of lymphocytic cells in bone marrow, spermatocytes in adult testes, and basal esophageal cells (4), raising the possibility that detection of DDR activation in vivo could allow early detection of precancerous and cancerous lesions (9).

It has been shown previously that an anti- $\gamma \mathrm{H} 2 \mathrm{AX}$ antibodybased imaging probe can detect $\gamma \mathrm{H} 2 \mathrm{AX}$ noninvasively (10). Anti$\gamma \mathrm{H} 2 \mathrm{AX}$ antibodies were modified by the addition of the HIV-1 transactivator of transcription protein-derived cell-penetrating peptide, transactivator of transcription (TAT), which harbors a nonclassic nuclear localization sequence, promoting nuclear translocation of the conjugate. Anti- $\gamma \mathrm{H} 2 \mathrm{AX}-\mathrm{TAT}$ was radiolabeled with ${ }^{111} \mathrm{In}$, to allow SPECT imaging. After the induction of $\gamma \mathrm{H} 2 \mathrm{AX}$ by $\mathrm{X}$-irradiation or bleomycin, ${ }^{111}$ In-anti- $\gamma \mathrm{H} 2 \mathrm{AX}-\mathrm{TAT}$ accumulated in human breast cancer xenografts in athymic mice, and the extent of ${ }^{111}$ In uptake was linearly dependent on the level of $\gamma \mathrm{H} 2 \mathrm{AX}$ expression (10).

In this report, the ability of the ${ }^{111}$ In-anti- $\gamma \mathrm{H} 2 \mathrm{AX}$-TAT probe to detect DDR during tumorigenesis was investigated using a transgenic mouse model of breast cancer in which the mouse mammary tumor virus (MMTV) promoter drives neuT expression. NeuT, a variant of the rat neu human epidermal growth factor receptor 2 homolog, contains a point mutation in the transmembrane region and is highly tumorigenic, compared with wild-type (WT) neu or human epidermal growth factor receptor 2 (11). When BALB-neuT mice reach $21-28 \mathrm{~d}$ of age, the neuT protein is overexpressed in mammary glands and areas of atypical hyperplasia start to form (12), which progress to in situ carcinomas at about day 60 and to invasive cancers by day 120-150. Neoplastic change occurs, albeit asynchronously, in all mammary glands so that by about $120 \mathrm{~d}, 1$ or more tumors are palpable and by about day 230 (33 wk) all 10 mammary glands contain palpable tumors (13). In this study, we 
explored the ability of ${ }^{111} \mathrm{In}-\mathrm{anti}-\gamma \mathrm{H} 2 \mathrm{AX}-\mathrm{TAT}$ SPECT imaging to detect tumors during oncogenesis in BALB-neuT mice.

\section{MATERIALS AND METHODS}

\section{Animal Model}

All animal procedures were performed in accordance with the U.K. Animals (Scientific Procedures) Act 1986 and with local ethical committee approval. Female WT BALB/c mice (Charles River) were crossed with male BALB-neuT mice (a gift from Guido Forni). The BALB-neuT strain originated from a transgenic $\mathrm{CD} 1$ random-bred breeder male mouse carrying the mutated rat neuT oncogene driven by the MMTV promoter (1). The mutated gene encodes a single-point mutation that replaces the valine residue at position 664 in the transmembrane domain of p185/neu with glutamic acid. This mutation promotes p185 homo- and heterodimerization and transforms the neu protooncogene into a dominant transforming oncogene. Offspring of the BALB-neuT male and WT female cross were genotyped using the following primers: $5^{\prime}$-CGCACGTCCTCCAGGTAG-3' and 5'-GATTCCAACGACCACCACTA-3'. NeuT-positive female mice were used for experiments.

\section{Immunohistochemistry}

At selected times, mammary tissue or mammary tumors were harvested from female BALB/c or BALB-neuT mice. Flash-frozen tissues were embedded in optimal-cutting-temperature (OCT) medium, and $10-\mu \mathrm{m}$ sections were prepared. Tissue sections were stained with hematoxylin and eosin, and images were acquired using a wide-field microscope. Alternatively, sections were washed to remove OCT medium, fixed (4\% paraformaldehyde, $10 \mathrm{~min}$, room temperature), permeabilized ( $1 \%$ triton X-100 in phosphate-buffered saline [PBS], $10 \mathrm{~min}$, room temperature), blocked ( $1 \%$ bovine serum albumin in $\left.\mathrm{PBS}, 1 \mathrm{~h}, 37^{\circ} \mathrm{C}\right)$, and incubated with anti- $\gamma \mathrm{H} 2 \mathrm{AX}$ antibodies raised in mouse (Calbiochem; 1:800 in blocking buffer, $1 \mathrm{~h}, 37^{\circ} \mathrm{C}$ ) or with anti-pATM antibodies raised in rabbit (R\&D Systems), followed by AlexaFluor488-labeled goat anti-mouse or AlexaFluor555-labeled goat anti-rabbit antibody (Invitrogen; 1:250 in blocking buffer, $1 \mathrm{~h}, 37^{\circ} \mathrm{C}$ ). Sections were mounted with Vectashield containing 4',6-diamidino-2-phenylindole to stain DNA (Vector Laboratories), and images were acquired using confocal microscopy (Zeiss 710; Carl Zeiss Ltd.). $\gamma \mathrm{H} 2 \mathrm{AX}$ foci were enumerated manually in more than 1,500 cells per sample, on more than 10 randomly selected view fields.

\section{In Vivo Imaging and Biodistribution}

${ }^{111}$ In-anti- $\gamma \mathrm{H} 2 \mathrm{AX}-\mathrm{TAT}$ and the nonspecific control probe ${ }^{111} \mathrm{In}-$ rabbitIgG-TAT ( $\left.{ }^{111} \mathrm{In}-\mathrm{rIgG}-\mathrm{TAT}\right)$ and the fluorophore-labeled probe Cy3-anti- $\gamma \mathrm{H} 2 \mathrm{AX}-\mathrm{TAT}$ were synthesized as reported (10). SPECT and CT images of BALB-neuT mice aged between 43 and $145 \mathrm{~d}$ were acquired under isoflurane anesthesia using a small-animal nanoSPECT/CT scanner (Bioscan) $24 \mathrm{~h}$ after intravenous administration of ${ }^{111} \mathrm{In}$-anti- $\gamma \mathrm{H} 2 \mathrm{AX}$-TAT (5 $\mu \mathrm{g}$; 16 animals) or ${ }^{111} \mathrm{In}$-rIgG-TAT (5 $\mu \mathrm{g}$; 16 animals). Imaging was repeated weekly for part of the period between the ages of 43 and $145 \mathrm{~d}$ per animal ( $5 \mathrm{MBq}$ of ${ }^{111}$ In per scan). Animals were euthanized when tumor size reached the permitted upper limit (12-mm gross median diameter). Volume-of-interest analysis of mammary fat pads and selected normal tissues was performed using the Inveon Research Workplace package (Siemens). ${ }^{111}$ In uptake in mammary fat pads and the age of animals were recorded. Tumors were scored as positive when the percentage injected dose per gram of tissue (\%ID/g) exceeded $5 \% \mathrm{ID} / \mathrm{g}$. ${ }^{111} \mathrm{In}$-anti- $\gamma \mathrm{H} 2 \mathrm{AX}$-TAT $(5 \mu \mathrm{g})$ was administered intravenously to BALB/c WT mice. After acquisition of SPECT images at $24 \mathrm{~h}$ after injection, mice were euthanized and organs were removed, weighed, and counted for radioactivity. To demonstrate $\gamma \mathrm{H} 2 \mathrm{AX}$ targeting in vivo, BALB-neuT or BALB/c WT mice aged 80-100 d (3 mice per group) received $\mathrm{Cy} 3$-anti- $\gamma \mathrm{H} 2 \mathrm{AX}-\mathrm{TAT}(5 \mu \mathrm{g})$ or PBS intravenously. At $24 \mathrm{~h}$ after injection, fluorescence imaging was performed, using an IVIS200 system (Perkin Elmer), as described previously (10). Mammary fat pads were removed, flash-frozen, sectioned, and processed for $\gamma \mathrm{H} 2 \mathrm{AX}$ immunostaining.

\section{MR Imaging}

T1-weighed and dynamic contrast-enhanced (DCE) MR imaging of BALB-neuT mice of different ages was performed before and $20 \mathrm{~min}$ after intravenous administration of gadodiamide (Omniscan; $15 \mu \mathrm{mol}$, 0.5 M [GE Healthcare]), using a dedicated small-animal MR imaging scanner. Data were collected using a T1-weighted, gradient and radiofrequency-spoiled, respiration-triggered, 3-dimensional gradient-echo sequence on a Varian 4.7-T MR imaging camera (Agilent). The following were imaging parameters: flip angle of $10^{\circ}$, repetition time of 2.06 $\mathrm{ms}$, echo time of $0.93 \mathrm{~ms}$, field of view of $54 \times 27 \times 27 \mathrm{~mm}^{3}$, isotropic resolution of $420 \mu \mathrm{m}$, and a temporal resolution of $11 \mathrm{~s}$. After acquisition of 10 frames, $15 \mu \mathrm{mol}$ gadodiamide were injected intravenously over $5 \mathrm{~s}$ via a catheter placed in a tail vein. Images were acquired for a further $20 \mathrm{~min}$. Semiquantitative gadolinium uptake parameters (area under the curve, first uptake moment) on a voxel-per-voxel basis were extracted for the whole tumor using MATLAB (The MathWorks) and parametric images generated. Images were inspected, and where tumors larger than $3 \times 3$ voxels were found, mice were scored as positive.

\section{Statistical Methods}

All data and graphs are expressed as mean $\pm \mathrm{SD}$, unless defined otherwise. Differences in the number of $\gamma \mathrm{H} 2 \mathrm{AX}$ foci per cell were assessed using an ANOVA test. Differences in the uptake of ${ }^{111} \mathrm{In}-$ anti- $\gamma \mathrm{H} 2 \mathrm{AX}$-TAT and ${ }^{111}$ In-rIgG-TAT were assessed by fitting a multilevel linear model with age, compound, and set (data were obtained in 2 independent sets of 20 and 12) as explanatory variables. An interaction between age and compound was fitted to allow different effects of age for each compound. The uptake of ${ }^{111} \mathrm{In}$-anti- $\gamma \mathrm{H} 2 \mathrm{AX}$-TAT and ${ }^{111} \mathrm{In}-$ rIgG-TAT in mice of each age group was compared by fitting a multilevel linear model. Age groups were chosen on the basis of known timing of development of mammary fat pad lesions in the BALB-neuT model. As a sensitivity analysis, nil measurements, for which no volume of interest could be drawn because no signal or structure was discernible, were excluded and the models were fitted again, but this variation did not alter the conclusion. Times to tumor detection by SPECT, MR imaging, and palpation were compared using log-rank tests. All statistical analyses were performed using the statistical software package R (14).

\section{RESULTS}

\section{DDR Activation During Carcinogenesis in BALB-neuT Mice}

Histologic examination of mammary fat pads from BALB-neuT mice confirmed the progressive development of atypical hyperplasia and in situ lesions, leading to invasive carcinoma by the age of 100$130 \mathrm{~d}$ (Fig. 1A). Normal duct structure was observed in mammary fat pads of mice aged up to $40 \mathrm{~d}$. By $75 \mathrm{~d}$, changes of atypical hyperplasia were noted consisting of dilated, irregular ducts with pleomorphic cells containing enlarged nuclei. At 90-95 d, there was extensive atypical hyperplasia with in situ carcinoma, typified by thickened ductal walls and small intraductal masses. Invasive carcinomas were apparent at 100-130 d (solid masses). In contrast, mammary fat pads from WT BALB/c mice aged $130 \mathrm{~d}$ showed normal histology (Supplemental Figs. 1A and 1B; supplemental materials are available at http://jnm.snmjournals.org). DDR activation, as indicated by the phosphorylation of $\mathrm{H} 2 \mathrm{AX}$ to form $\gamma \mathrm{H} 2 \mathrm{AX}$, was observed in hyperplastic lesions and ductal carcinoma in situlike mammary fat pad lesions in BALB-neuT mice at 75-95 d (Fig. 1A). Also, although staining for total ATM in mammary fat pads/ tumors was positive, with low-level expression at all times tested, the activated form (pATM) of the enzyme was clearly present only at 75-95 d, further evidence of upregulation of the DDR during this 


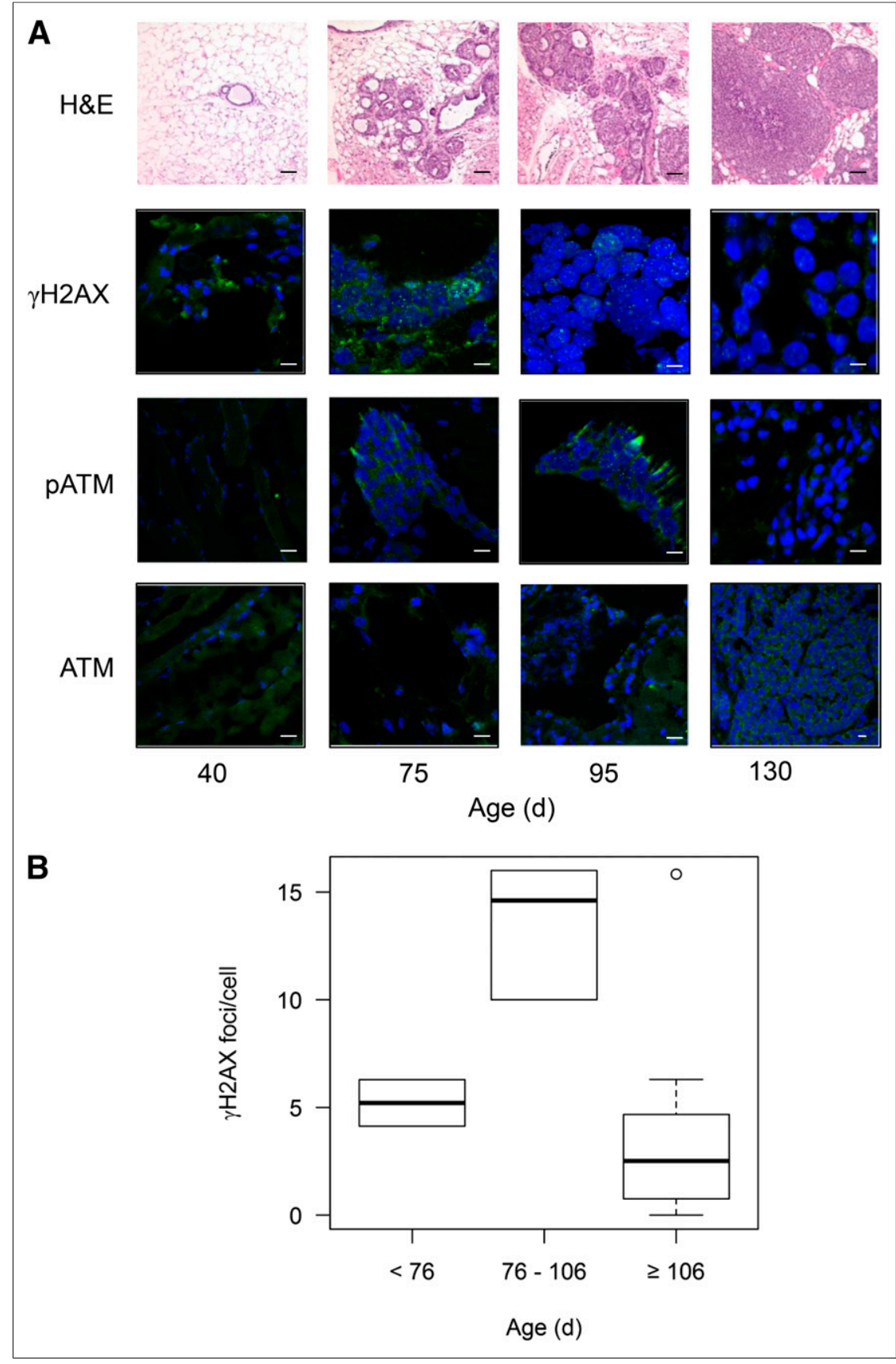

FIGURE 1. Mammary fat pads or mammary fat pad tumors were harvested from BALB-neuT mice of various ages. (A) Tissues were frozen, sectioned, and stained with hematoxylin and eosin (H\&E) or immunostained for yH2AX, pATM, or ATM. Representative images are shown (scale bar $=10 \mu \mathrm{m}$ ). (B) Number of $\mathrm{yH} 2 \mathrm{AX}$ foci per cell in harvested tissues was counted (1 outlier depicted in gray). ${ }^{\star} P<0.05$.

phase. The number of $\gamma \mathrm{H} 2 \mathrm{AX}$ foci was greater in hyperplastic lesions in mammary fat pads from mice aged $76-106 \mathrm{~d}$ than in mammary fat pads from mice aged less than $75 \mathrm{~d}(13.5 \pm 1.2$ vs. $5.2 \pm 1.5$ foci/cell; $P<0.05)$ and in carcinomas from mice aged older than $106 \mathrm{~d}(13.5 \pm$ 1.2 vs. $3.4 \pm 1.1$ foci/cell; $P<0.001$ ) (Fig. 1B).

\section{Targeting yH2AX In Vivo}

To confirm that systemically delivered anti- $\gamma \mathrm{H} 2 \mathrm{AX}$-TAT probe colocalizes with $\gamma \mathrm{H} 2 \mathrm{AX}$ in vivo, fluorescence imaging of BALB$n e u \mathrm{~T}$ or $\mathrm{BALB} / \mathrm{c}$ WT mice (age, $80-100 \mathrm{~d}$ ) was performed
$24 \mathrm{~h}$ after intravenous administration of Cy3anti- $\gamma \mathrm{H} 2 \mathrm{AX}-\mathrm{TAT}$. The fluorescent signal localized in mammary fat pads in BALBneuT mice (Fig. 2A). No fluorescent signal was observed in fat pads of $\mathrm{BALB} / \mathrm{c}$ mice or in BALB-neuT mice that received PBS intravenously. A feature of the BALB-neuT model is the development of lymph node metastases. Also, whereas the neuT oncogene is mainly expressed in mammary glands, it is expressed to a lesser extent in salivary glands. Therefore, some of the areas of uptake may represent lymph node or salivary gland deposits. Mammary tissue was harvested and immunostained for $\gamma \mathrm{H} 2 \mathrm{AX}$. Colocalization of Cy3-anti- $\gamma \mathrm{H} 2 \mathrm{AX}-\mathrm{TAT}$ and $\gamma \mathrm{H} 2 \mathrm{AX}$ foci was observed (Fig. 2B).

\section{${ }^{111}$ In-Anti-yH2AX-TAT SPECT Imaging}

To study $\gamma \mathrm{H} 2 \mathrm{AX}$ expression, as a marker of DDR activation, throughout the progression of mammary fat pad lesions, BALB-neuT mice were imaged weekly using SPECT, $24 \mathrm{~h}$ after intravenous administration of ${ }^{111} \mathrm{In}-$ anti- $\gamma \mathrm{H} 2 \mathrm{AX}$-TAT. The data consist of 148 observations on 32 animals, obtained in 2 sets of 20 and 12 (Figs. 3A-3C). Restriction on the number of weekly scans each animal underwent meant that no single animal was imaged throughout the entire period of interest (from 40 to $130 \mathrm{~d}$ ). However, a complete time course that includes measurements from all mice is presented (Fig. 3C). Plots for individual animals for which at least 4 observations were made are shown in Supplemental Fig. 2. The uptake of ${ }^{111}$ In-anti- $\gamma \mathrm{H} 2 \mathrm{AX}$-TAT was higher than that of ${ }^{111} \mathrm{In}$-rIgG-TAT $(P=0.0038$; adjusted for age and set). Intratumoral accumulation of both ${ }^{111} \mathrm{In}$-anti- $\gamma \mathrm{H} 2 \mathrm{AX}-\mathrm{TAT}$ and ${ }^{111}$ In-rIgG-TAT was high in older mice (>106 d), compared with younger mice, suggesting that nonspecific enhanced permeability and retention-mediated uptake of IgG-TAT constructs occurs in mature tumors. This enhancement may be attributable to leakiness of vessels in large, fully established tumors. In mice aged less than $76 \mathrm{~d}$, tumor uptake of ${ }^{111}$ In-anti- $\gamma \mathrm{H} 2 \mathrm{AX}$ TAT and ${ }^{111}$ In-rIgG-TAT did not differ significantly $(2.52 \pm 2.02$ vs. $1.42 \pm 1.72$ $\% \mathrm{ID} / \mathrm{g}$, respectively). This result was consistent with the multilevel model in which the coefficient for ${ }^{111} \mathrm{In}$-anti- $\gamma \mathrm{H} 2 \mathrm{AX}$-TAT was estimated to be $1.19 \pm 0.92, P=0.24$. That is, for the ${ }^{111}$ In-anti$\gamma \mathrm{H} 2 \mathrm{AX}$-TAT group the uptake for ages more than $76 \mathrm{~d}$ is expected to be 1.19 higher than in the ${ }^{111} \mathrm{In}$-rIgG-TAT group. However, ${ }^{111} \mathrm{In}$ anti- $\gamma \mathrm{H} 2 \mathrm{AX}-\mathrm{TAT}$ accumulated to a greater extent than ${ }^{111} \mathrm{In}-\mathrm{rIgG}-$ TAT in the mammary fad pads of mice aged 76-106 d (3.67 \pm 3.01 vs. $1.48 \pm 1.86 \% \mathrm{ID} / \mathrm{g}$, respectively; intercept, $1.60 \pm 0.59$; and coefficient $[\beta]$ for ${ }^{111}$ In-anti- $\gamma \mathrm{H} 2 \mathrm{AX}$-TAT, $2.22 \pm 0.80 ; P=0.01$ for $\beta$ ), which corresponds to the development of in situ and early 


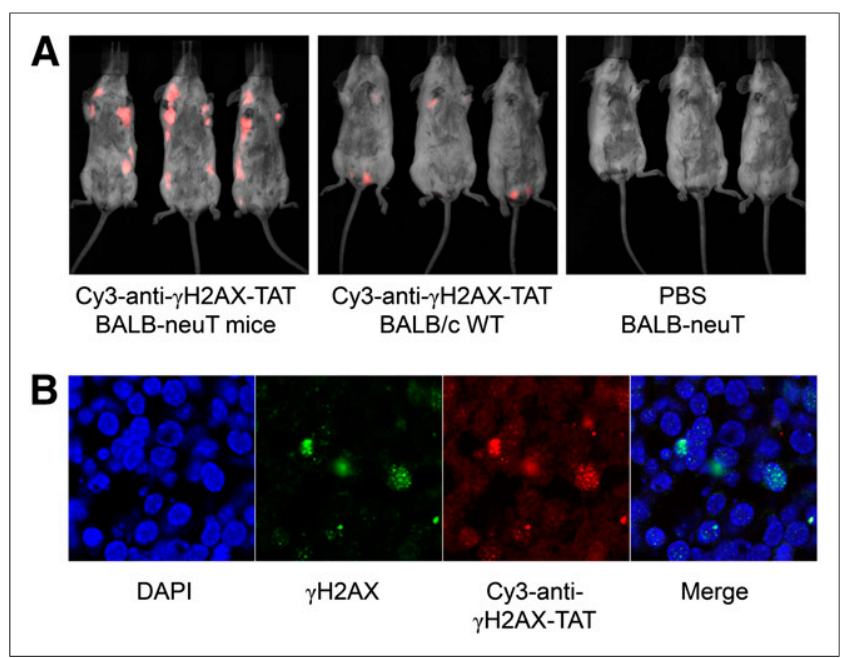

FIGURE 2. (A) Groups of 3 BALB-neuT or BALB/c WT mice aged 76106 d received Cy3-anti-yH2AX-TAT or PBS intravenously. Fluorescence images were acquired at $24 \mathrm{~h}$ after injection. (B) Mammary fat pads were removed, frozen, sectioned, and stained for $y \mathrm{H} 2 \mathrm{AX}$ and counterstained with DAPI to visualize nucleus (blue). Colocalization of $\mathrm{yH} 2 \mathrm{AX}$ (green) and Cy3-anti-yH2AX-TAT (red) is observed (scale bar = $10 \mu \mathrm{m})$. DAPI $=$ 4',6-diamidino-2-phenylindole.

invasive carcinomas. For this group, ${ }^{111} \mathrm{In}$-anti- $\gamma \mathrm{H} 2 \mathrm{AX}-\mathrm{TAT}$ uptake often exceeded $5 \% \mathrm{ID} / \mathrm{g}$, whereas in only 1 case did the uptake of the control probe marginally exceed $5 \% \mathrm{ID} / \mathrm{g}$. Additionally, analysis of data on individual lesions showed that all lesions that became palpable during the study had been identified by SPECT imaging before becoming detectable by palpation. In 14 of 16 mice, the first lesion to be detected by ${ }^{111} \mathrm{In}$-anti- $\gamma \mathrm{H} 2 \mathrm{AX}$-TAT SPECT imaging was subsequently also the first lesion to become palpable.

${ }^{111} \mathrm{In}$-anti- $\gamma \mathrm{H} 2 \mathrm{AX}-\mathrm{TAT}$ was administered to young or old BALB/c WT mice, and after acquisition of SPECT images at $24 \mathrm{~h}$ tissues were retrieved for radioactivity counting (Supplemental Fig. 3). The uptake of ${ }^{111}$ In-anti- $\gamma \mathrm{H} 2 \mathrm{AX}$-TAT was slightly higher in the mammary fat pads of young mice than old mice; however, this was not statistically significant (mean $\pm \mathrm{SD}, 2.28 \pm 0.35$ vs. $1.21 \pm 0.49$; $P=0.10)$. The level of uptake in the mammary fat pads in BALB/c WT mice was therefore well below the $5 \% \mathrm{ID} / \mathrm{g}$ cutoff that was used to denote positive ${ }^{111}$ In-anti- $\gamma \mathrm{H} 2 \mathrm{AX}$-TAT uptake in BALBneu T mice.

\section{Lack of Effect of ${ }^{111}$ In-Radioimmunoconjugates on yH2AX Expression and Tumor Growth}

To evaluate the possibility that administration of ${ }^{111}$ In-anti$\gamma \mathrm{H} 2 \mathrm{AX}-\mathrm{TAT}$ or ${ }^{111} \mathrm{In}$-rIgGTAT could itself affect tumor development, the tumor-free survival of mice that had undergone repeated imaging was compared with that of control animals. No significant differences were detected (log-rank test; $P=0.41$ ) (Fig. 4). Similarly, in a group of animals aged 89-96 d there was no statistical difference in the number of $\gamma \mathrm{H} 2 \mathrm{AX}$ foci per tumor cell in mice that received ${ }^{111} \mathrm{In}$-anti- $\gamma \mathrm{H} 2 \mathrm{AX}$-TAT, compared with those that did not. The mean $\gamma \mathrm{H} 2 \mathrm{AX}$ foci per cell $24 \mathrm{~h}$ after a single intravenous injection of ${ }^{111} \mathrm{In}$-anti- $\gamma \mathrm{H} 2 \mathrm{AX}$-TAT was $14.7 \pm 3.1$ $(n=12)$ versus $13.5 \pm 2.9(n=6)$ for controls $(P=0.65)$.

\section{Early Detection of Precancerous Lesions}

Small tumors were not identified in CT images, acquired to provide an anatomic reference for SPECT images (results not shown). However, tumors with a diameter as small as $1.26 \mathrm{~mm}$ were visualized using T1-weighed MR images and were particularly clearly identifiable in area-under-the-curve parameter maps generated after DCE MR imaging (Fig. 5A). The median age at which tumors were detectable by DCE MR imaging and palpation was 120 and $131 \mathrm{~d}$, respectively $(P=0.10)$. SPECT imaging using ${ }^{111}$ In-anti- $\gamma \mathrm{H} 2 \mathrm{AX}-\mathrm{TAT}$ was superior to DCE MR imaging $(P=0.058)$, with median age at detection of first tumor of $96 \mathrm{~d}$ (Fig. 5B).

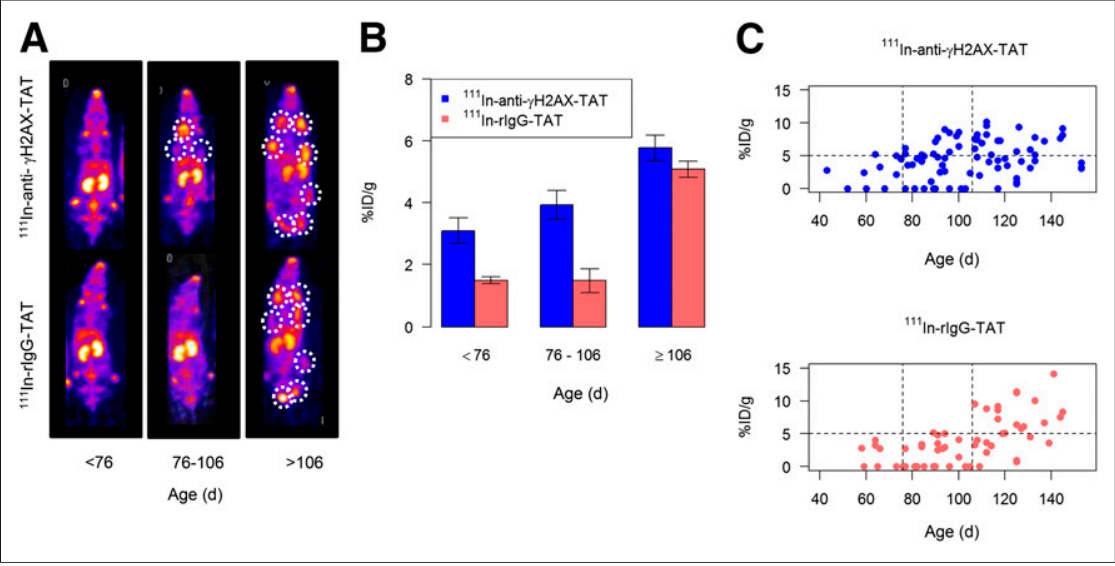

FIGURE 3. BALB-neuT mice of different ages received ${ }^{111} \mathrm{In}$-anti-yH2AX-TAT or ${ }^{111} \mathrm{In}$-rlgGTAT intravenously at weekly intervals for up to $5 \mathrm{wk}$. SPECT images were acquired $24 \mathrm{~h}$ after injection. (A) Maximum-intensity projections of representative animals. White circles denote areas of tumor development. (B) Uptake of ${ }^{111} \mathrm{In}$ in mammary tissue was quantified by volume-of-interest analysis. For ${ }^{111} \mathrm{In}$-anti-yH2AX-TAT or ${ }^{111} \mathrm{In}$-rlgGTAT observations were assigned to 3 groups by age, and then within each age group the average observation for each mouse was calculated. Height of each bar represents mean of these averaged observations, and error bars are \pm SEM. ${ }^{*} P<$ 0.05. (C) Uptake of ${ }^{111} \mathrm{In}$ in mammary tissue was quantified by volume-of-interest analysis. For ${ }^{111} \mathrm{In}$-anti-yH2AX-TAT or ${ }^{111} \mathrm{In}$-rlgG-TAT, observations from individual mice were plotted against age of mice at time of observation. Age grouping is indicated by the vertical dashed lines. Horizontal dashed line indicates $5 \% \mathrm{ID} / \mathrm{g}$ threshold above which uptake was designated as positive.

\section{DISCUSSION}

Oncogenic stimulation leading to DNA replication stress occurs early in the development of cancer. The resulting accumulation of DNA damage triggers constitutive activation of the DDR, typified by the expression of phosphorylated forms of key DNA repair proteins including pATM (S1981), $\gamma \mathrm{H} 2 \mathrm{AX}$ (S139), and pChk2 (Thr68) (3,5,9, 15,16). As a result of DDR activation, DNA damage is repaired and its detrimental effects, including genomic instability, avoided. However, unrelieved oncogenic stress eventually results in a breach of this protective mechanism with subsequent progression to the malignant phenotype. Extensive published evidence has shown that the DDR is activated during development of many tumor types (17). Several reports have shown immunohistochemical evidence that DDR proteins are highly expressed in precancerous lesions but less so in invasive cancers or corresponding normal tissues (4-8). It is therefore logical to explore these molecules 


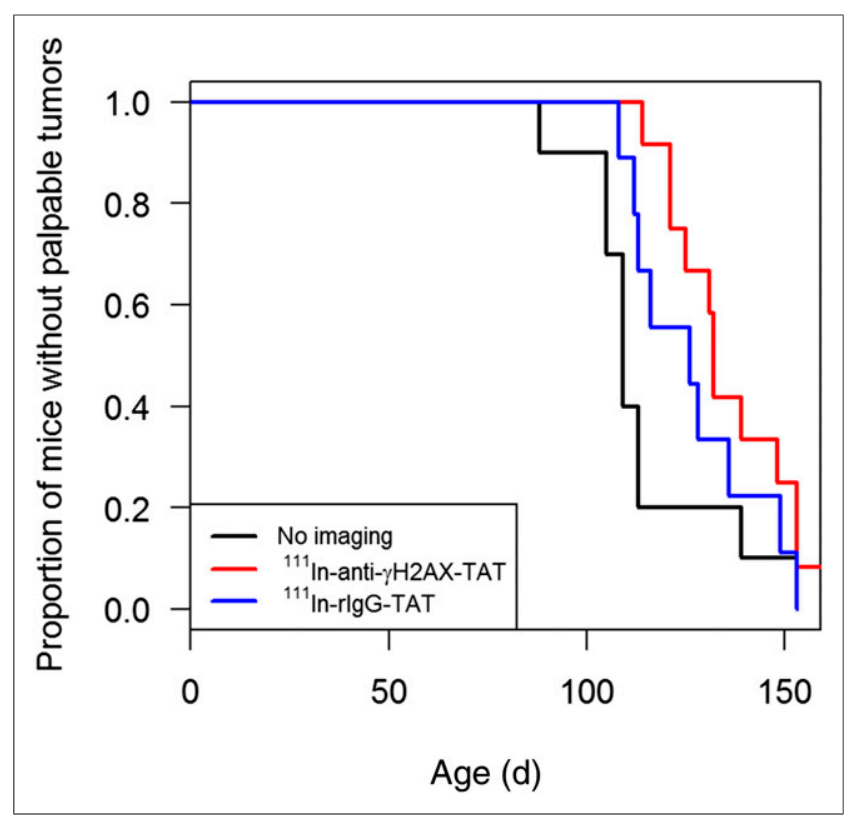

FIGURE 4. Kaplan-Meier plot comparing tumor-free survival of control BALB-neuT mice (that did not receive imaging probes) with that of mice that were imaged on multiple occasions using ${ }^{111} \mathrm{In}$-anti-yH2AX-TAT or ${ }^{111}$ In-rlgG-TAT.

as biomarkers of early cancer development and reasonable to hypothesize that visualization of components of the DDR might provide an excellent means of early cancer detection (9). It was shown previously that it is possible to image DNA damage using the prototypic SPECT imaging tracer ${ }^{111}$ In-anti- $\gamma \mathrm{H} 2 \mathrm{AX}-\mathrm{TAT}$ (10). We have used the same probe to test the hypothesis that DDR imaging can detect premalignant lesions in the BALB-neuT breast cancer model.

The expression of $\gamma \mathrm{H} 2 \mathrm{AX}$ was significantly increased in hyperplastic and ductal carcinoma in situ lesions in mice aged between 76 and $106 \mathrm{~d}$, compared with normal mammary fat pads (from young mice) and mature tumors (from older mice) (Fig. 1B). pATM, the kinase primarily responsible for $\mathrm{H} 2 \mathrm{AX}$ phosphorylation to form $\gamma \mathrm{H} 2 \mathrm{AX}$, was also upregulated during this period, whereas total ATM remained unchanged. In contrast to this robust activation of the DDR in BALBneu $\mathrm{T}$-associated preneoplastic lesions, Reddy et al. found that the DDR was either weak or absent in hyperplastic lesions arising in some other transgenic mouse models of breast cancer. For example, only modest induction of $\gamma \mathrm{H} 2 \mathrm{AX}$ was observed in mammary lesions from MMTV-ErbB2 transgenic animals (18). One possible explanation for this difference is that NeuT is more potently oncogenic than ErbB2 (11) and so is more likely to cause DDR activation. Interestingly, a somatic model of breast carcinogenesis, consisting of intraductal injection of the avian retrovirus RCAS carrying ErbB2 in MMTVtva mice, was associated with marked stimulation of the DDR (18).

Immunofluorescence staining for $\gamma \mathrm{H} 2 \mathrm{AX}$ in tumors from mice that had received $\mathrm{Cy} 3$-anti- $\gamma \mathrm{H} 2 \mathrm{AX}$-TAT intravenously showed good colocalization of $\mathrm{Cy} 3$ with $\gamma \mathrm{H} 2 \mathrm{AX}$ foci, indicating that systemically administered antibody tracer is capable of penetrating tumor, internalizing into cancer cells and associating with its intranuclear target. Serial SPECT imaging using ${ }^{111}$ In-anti- $\gamma \mathrm{H} 2 \mathrm{AX}-\mathrm{TAT}$ in BALB-neuT mice showed that the $\gamma \mathrm{H} 2 \mathrm{AX}$ signal was greater in mammary fat pads of mice aged 76-106 $d$ than in the pads of animals in younger and older age groups. This greater signal was consistent with ex vivo immunostaining of mammary fat pads that revealed that $\gamma \mathrm{H} 2 \mathrm{AX}$ expression was highest in 76- to 106-d-old mice.
It was important to investigate whether ${ }^{111}$ In-anti- $\gamma \mathrm{H} 2 \mathrm{AX}-\mathrm{TAT}$ itself increases DNA double-strand breaks, either through radiation dose deposition or by blocking signaling downstream of H2AX. However, we saw no evidence that ${ }^{111} \mathrm{In}$-anti- $\gamma \mathrm{H} 2 \mathrm{AX}$-TAT perturbs the system it is designed to image. Specifically, the number of $\gamma \mathrm{H} 2 \mathrm{AX}$ foci in tumor cells was unaffected by exposure to the probe, and repeated administration of ${ }^{111}$ In-anti- $\gamma \mathrm{H} 2 \mathrm{AX}$-TAT or ${ }^{111} \mathrm{In}-\mathrm{rIgG}$ TAT had no effect on the tumor-free survival of animals (Fig. 4).

${ }^{111}$ In-anti- $\gamma \mathrm{H} 2 \mathrm{AX}-\mathrm{TAT}$ SPECT imaging of the DDR was superior to anatomic imaging methods. CT, acquired as anatomic reference during SPECT, provided images with poor soft-tissue contrast such that only tumors greater than $5 \mathrm{~mm}$ in diameter were detectable. T1-weighed MR imaging and DCE MR imaging provided better contrast. However, the average age of mice when tumors first became detectable by DCE MR imaging was $120 \mathrm{~d}$, significantly later than detection by DDR imaging using SPECT (i.e., $96 \mathrm{~d}$ ).

The data presented in Figure 3 show that uptake of both the ${ }^{111} \mathrm{In}$ anti- $\gamma \mathrm{H} 2 \mathrm{AX}-\mathrm{TAT}$ and the nonspecific probe is higher in carcinomas in older mice than in intermediate-aged mice, raising the question as to how the extent of uptake of ${ }^{111} \mathrm{In}$-anti- $\gamma \mathrm{H} 2 \mathrm{AX}$-TAT could be interpreted in a practical setting. Because SPECT is usually combined with anatomic imaging such as CT or MR imaging, it is possible that late-stage lesions would be detectable by both correlative imaging and ${ }^{111} \mathrm{In}$-anti- $\gamma \mathrm{H} 2 \mathrm{AX}$-TAT whereas preneoplastic

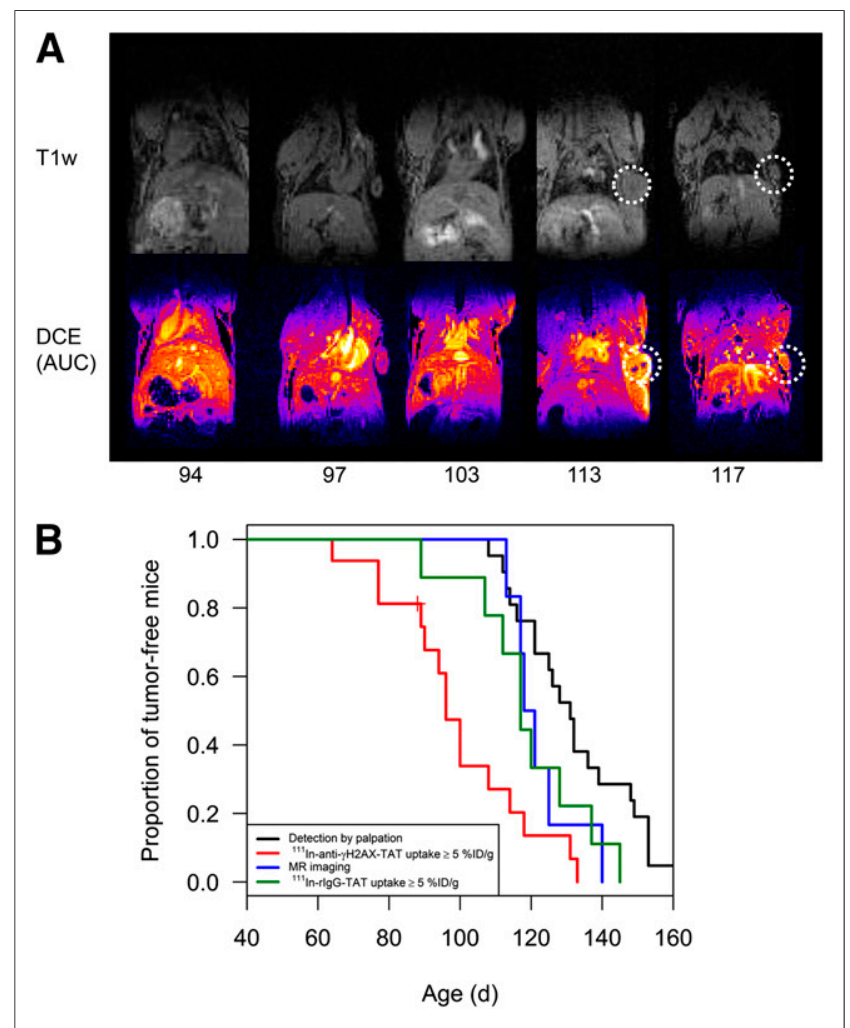

FIGURE 5. (A) T1-weighed (T1w) images of BALB-neuT mice of varying ages were acquired on 4.7-T MR imaging scanner. DCE MR imaging: repeated T1-weighed images were acquired after injection of gadodiamide, and integrated signal enhancement (area under the curve [AUC]) was calculated on pixel-per-pixel basis. (B) Kaplan-Meier plot comparing age at detection of precancerous lesions and tumors through palpation, DCE MR imaging, all SPECT imaging $\left({ }^{111} / n\right.$-antiyH2AX-TAT or ${ }^{111}$ In-rlgG-TAT), and ${ }^{111}$ In-anti-yH2AX-TAT SPECT designated as positive when uptake of ${ }^{111} \mathrm{In}$-anti-yH2AX-TAT was $\geq 5 \% \mathrm{ID} / \mathrm{g}$. 
lesions would be positive for ${ }^{111}$ In-anti- $\gamma \mathrm{H} 2 \mathrm{AX}$-TAT but possibly lack CT or MR imaging evidence of a mass lesion. Although anatomic imaging might help in this way, it is clear that more detailed analysis of the pattern of uptake of ${ }^{111} \mathrm{In}$-anti- $\gamma \mathrm{H} 2 \mathrm{AX}$-TAT during progression from preneoplasia to invasion is needed.

These results provide proof of principle that it is possible to image $\gamma \mathrm{H} 2 \mathrm{AX}$ noninvasively in preneoplastic lesions. However, further optimization of ${ }^{111} \mathrm{In}$-anti- $\gamma \mathrm{H} 2 \mathrm{AX}$-TAT is needed, because the ratio of tumor to normal tissue contrast achieved in SPECT images was modest. The investigation of other tumor models is needed to explore the generalizability of these findings. Further work is also required to determine whether all lesions with constitutive DDR activation are destined to become invasive cancers as this activation is likely to be a significant determinant of the utility of this imaging approach. It is possible that the DDR is particularly prominent in senescent premalignant cells, which may or may not progress beyond metaplasia, because the DDR itself acts as a barrier to tumorigenesis (19). Imaging $\gamma \mathrm{H} 2 \mathrm{AX}$ provides no information about other modifications of H2AX, such as ubiquitination and SUMOylation that may also follow genotoxic insults and be functionally important.

A concerted effort is being made to develop imaging biomarkers for the detection of preneoplasia in human cancers. The application of advanced endoscopic techniques to the diagnosis of premalignant lesions of the upper aerodigestive tract, bladder, colon, esophagus, and bronchi is a major focus of research $(20,21)$. For example, confocal laser endomicroscopy has recently been confirmed to significantly improve the identification of dysplasia in Barrett's esophagus when combined with high-definition white light endoscopy (22). Optical and fluorescence endoscopic imaging techniques hold promise as accurate methods for distinguishing preneoplastic from invasive lesions, but visualization is limited to a depth of a few millimeters or less, and so they are not suitable for the diagnosis of endoscopically inaccessible or deep-seated lesions. Increasingly, the merits of functional MR imaging for the detection and characterization of precancerous lesions in the breast are being explored. For example, diffusion-weighted imaging helped differentiate high-risk breast lesions requiring further investigation from benign lesions (23). However, the specificity of these methods is not yet sufficient for widespread adoption in the clinic, and further research is required to develop clinically robust imaging systems for the detection of breast preneoplasia.

The approach of DNA damage imaging has potential applications in the early detection of cancer and screening cancer-prone individuals. Also the ability to image the DDR noninvasively would enable the response to DDR-targeted drugs to be monitored and might be useful in the development of cancer prevention strategies. Also, because DDR activation is a common phenomenon during tumorigenesis, $\gamma \mathrm{H} 2 \mathrm{AX}$-targeted molecular imaging may be applicable to early detection of several types of cancer.

\section{CONCLUSION}

DDR imaging using ${ }^{111}$ In-anti- $\gamma \mathrm{H} 2 \mathrm{AX}$-TAT identified mammary tumors significantly earlier than MR imaging. Imaging the DDR holds promise for the detection of preneoplasia and as a technique for screening cancer-prone individuals.

\section{DISCLOSURE}

The costs of publication of this article were defrayed in part by the payment of page charges. Therefore, and solely to indicate this fact, this article is hereby marked "advertisement" in accordance with 18 USC section 1734. This work was supported by the Medical Research Council, Cancer Research U.K. and by the Cancer Research U.K./Engineering and Physical Sciences Research Council Oxford Cancer Imaging Centre. No other potential conflict of interest relevant to this article was reported.

\section{REFERENCES}

1. Thompson LH. Recognition, signaling, and repair of DNA double-strand breaks produced by ionizing radiation in mammalian cells: the molecular choreography. Mutat Res. 2012;751:158-246.

2. Li X, Xu H, Xu C, et al. The yin-yang of DNA damage response: roles in tumorigenesis and cellular senescence. Int J Mol Sci. 2013;14:2431-2448.

3. Bartkova J, Rezaei N, Liontos M, et al. Oncogene-induced senescence is part of the tumorigenesis barrier imposed by DNA damage checkpoints. Nature. 2006; 444:633-637.

4. Bartkova J, Bakkenist CJ, Rajpert-De Meyts E, et al. ATM activation in normal human tissues and testicular cancer. Cell Cycle. 2005;4:838-845.

5. Gorgoulis VG, Vassiliou LV, Karakaidos P, et al. Activation of the DNA damage checkpoint and genomic instability in human precancerous lesions. Nature. 2005;434: 907-913.

6. Koorstra JB, Hong SM, Shi C, et al. Widespread activation of the DNA damage response in human pancreatic intraepithelial neoplasia. Mod Pathol. 2009;22: 1439-1445.

7. Kshirsagar M, Jiang W, Shih Ie M. DNA damage response is prominent in ovarian high-grade serous carcinomas, especially those with Rsf-1 (HBXAP) overexpression. J Oncol. 2012;2012:621685.

8. Matsuda Y, Wakai T, Kubota M, et al. DNA damage sensor gamma-H2AX is increased in preneoplastic lesions of hepatocellular carcinoma. ScientificWorldJournal. 2013;2013:597095.

9. Halazonetis TD, Gorgoulis VG, Bartek J. An oncogene-induced DNA damage model for cancer development. Science. 2008;319:1352-1355.

10. Cornelissen B, Kersemans V, Darbar S, et al. Imaging DNA damage in vivo using gammaH2AX-targeted immunoconjugates. Cancer Res. 2011;71:45394549 .

11. Quaglino E, Mastini C, Forni G, Cavallo F. ErbB2 transgenic mice: a tool for investigation of the immune prevention and treatment of mammary carcinomas. Curr Protoc Immunol. 2008; Chapter 20:Unit 20.9.1-9.10.

12. Di Carlo E, Diodoro MG, Boggio K, et al. Analysis of mammary carcinoma onset and progression in HER-2/neu oncogene transgenic mice reveals a lobular origin. Lab Invest. 1999;79:1261-1269.

13. Calogero RA, Cordero F, Forni G, Cavallo F. Inflammation and breast cancer: inflammatory component of mammary carcinogenesis in ErbB2 transgenic mice. Breast Cancer Res. 2007;9:211.

14. R Core Team. R: a language and environment for statistical computing. R Foundation for Statistical Computing. The R Project for Statistical Computing website. http://www.R-project.org/.

15. Bartek J, Lukas J, Bartkova J. DNA damage response as an anti-cancer barrier: damage threshold and the concept of 'conditional haploinsufficiency.' Cell Cycle. 2007;6:2344-2347.

16. Bartek J, Bartkova J, Lukas J. DNA damage signalling guards against activated oncogenes and tumour progression. Oncogene. 2007;26:7773-7779.

17. Bartkova J, Horejsi Z, Koed K, et al. DNA damage response as a candidate anticancer barrier in early human tumorigenesis. Nature. 2005;434:864-870.

18. Reddy JP, Peddibhotla S, Bu W, et al. Defining the ATM-mediated barrier to tumorigenesis in somatic mammary cells following ErbB2 activation. Proc Natl Acad Sci USA. 2010;107:3728-3733.

19. Campisi J, d'Adda di Fagagna F. Cellular senescence: when bad things happen to good cells. Nat Rev Mol Cell Biol. 2007;8:729-740.

20. Vakoc BJ, Fukumura D, Jain RK, Bouma BE. Cancer imaging by optical coherence tomography: preclinical progress and clinical potential. Nat Rev Cancer. 2012;12:363-368.

21. Subramanian V, Ragunath K. Advanced endoscopic imaging: a review of commercially available technologies. Clin Gastroenterol Hepatol. 2014;12:368376.

22. Sharma P, Meining AR, Coron E, et al. Real-time increased detection of neoplastic tissue in Barrett's esophagus with probe-based confocal laser endomicroscopy: final results of an international multicenter, prospective, randomized, controlled trial. Gastrointest Endosc. 2011;74:465-472.

23. Parsian S, Rahbar H, Allison KH, et al. Nonmalignant breast lesions: ADCs of benign and high-risk subtypes assessed as false-positive at dynamic enhanced MR imaging. Radiology. 2012;265:696-706. 